\title{
Le continent logique. À propos de Quadratura americana d'Emmanuel Désveaux
}

\section{Klaus Hamberger}

\section{OpenEdition}

1 Journals

Édition électronique

URL : https://journals.openedition.org/jsa/1444

DOI : 10.4000/jsa. 1444

ISSN : 1957-7842

\section{Éditeur}

Société des américanistes

\section{Édition imprimée}

Date de publication : 5 juin 2004

Pagination : 103-114

ISSN : 0037-9174

\section{Référence électronique}

Klaus Hamberger, "Le continent logique. À propos de Quadratura americana d'Emmanuel

Désveaux », Journal de la Société des américanistes [En ligne], 90-2 | 2004, mis en ligne le 10 septembre 2014, consulté le 06 septembre 2022. URL : http://journals.openedition.org/jsa/1444 ; DOI : https:// doi.org/10.4000/jsa. 1444 


\title{
POSITION
}

\section{LE CONTINENT LOGIQUE \\ À PROPOS DE QUADRATURA AMERICANA \\ D'EMMANUEL DÉSVEAUX *}

\author{
Klaus HAMBERGER **
}

Cet ouvrage d'Emmanuel Désveaux est une étude de logique transformationnelle appliquée à la pensée amérindienne. Cette pensée est supposée, par l'auteur; être l'objet privilégié, voire exclusif, d'une telle analyse. Les Mythologiques de Lévi-Strauss forment donc évidemment l'arrière-fond intellectuel de cette étude. En dépit de cela, son sous-titre, Essai d'anthropologie lévi-straussienne, risque de tromper le lecteur, d'une part, parce qu'il s'agit de bien plus qu'une simple continuation de la démarche lévi-straussienne, d'autre part, parce que les divergences avec cette démarche - notamment sur des points fondamentaux - sont nombreuses, comme l'auteur lui-même le souligne.

Dans ce livre, Désveaux développe essentiellement trois projets que nous allons discuter l'un après l'autre, bien qu'ils soient poursuivis parallèlement dans la plupart des chapitres. Premièrement, étendre la méthode transformationnelle que Lévi-Strauss avait délibérément restreinte au domaine mythologique à d'autres formes symboliques telles que le rite, la culture matérielle, les organisations sociales et les terminologies de parenté. Deuxièmement, caractériser ce champ transformationnel par des structures invariantes et isoler des traits distinctifs de la pensée amérindienne, pensée qui fonctionnerait de façon très différente de celles présentes dans d'autres grandes aires culturelles. Troisièmement, clarifier l'aspect formel des opérations de transformation symbolique agissant sur des systèmes d'oppositions binaires et tourner la "formule canonique » lévistraussienne en un véritable instrument analytique comparable aux formules d'algèbre.

Avant d'entrer dans le détail de ces trois volets du programme de recherche, signalons d'ores et déjà que celui-ci aboutit à une conclusion qui est non seulement différente, mais diamétralement opposée à certaines idées cru-

* Désveaux Emmanuel, Quadratura americana. Essai d'anthropologie lévi-straussienne, Genève, Georg Éditeurs, 2001, 641 p., bibliogr., index, ill., photos, fig., tabl., coll. « Ethnos ».

** Laboratoire d'anthropologie sociale, Paris [klaus_hamberger@yahoo.fr].

Journal de la Société des Américanistes, 2004, 90-2, pp. 103-114. OSociété des Américanistes. 
ciales que Lévi-Strauss avait maintenues depuis Les Structures élémentaires de la parenté (1949) jusqu'à Histoire de Lynx (1991) : le principe de réciprocité ne serait pas fondateur pour les organisations sociales (et notamment matrimoniales) des Amérindiens. Plus encore: la pensée amérindienne, en raison même de son caractère transformationnel qui entraînerait justement sa clôture, serait fondamentalement hostile à toute forme de communication impliquant la reconnaissance d'autrui. Cette connexion intime entre la pensée en transformations et la négation de l'altérité - caractéristique d'une culture non pas échangiste mais prédatrice - constitue en effet la thèse centrale de ce livre.

Pour la démontrer, l'auteur exploite et compare un vaste matériel d'ethnographie, provenant pour la plupart de l'Amérique du Nord et, surtout, de sa partie septentrionale (le Canada et l'Alaska); partie que l'auteur connaît bien puisqu'il a fait son terrain chez les Ojibwa septentrionaux et pour laquelle il fait les analyses les plus minutieuses dans cet ouvrage. On retiendra en particulier celles élaborées pour les métamorphoses des édicules rituels depuis le Labrador jusqu'au Plateau (chapitres III et IV), pour les masques et coiffures de la Côte Nord-Ouest jusqu'au Nord-Est, en passant par l'aire Inuit (chapitres XVIII et $\mathrm{XIX)}$, enfin pour les systèmes claniques algonkin, iroquois et sioux (chapitre XXIV). À ces études détaillées s'ajoutent des travaux comparatistes plus sommaires, traitant, entre autres, du jeu de balle dans tout l'Est du sous-continent (chapitre XII), des organisations sociales de la zone subarctique, sur la Côte Nord-Ouest, dans les Plaines et chez les Pueblo (chapitre XIII) et du champ énormément différencié des nomenclatures de parenté nord-américaines (chapitres XXII et XXIII) dont la discussion - hormis la terminologie Ojibwa - n'est certainement qu'une esquisse prometteuse d'un autre ouvrage sur ce thème. Enfin, trois études plutôt monographiques sont consacrées aux Natchez (chapitres XIV et XV), aux Inuit (chapitre XVII) et aux Miwok (chapitre XXV). L'Amérique du Sud n'apparaît en général qu'en comparaison directe avec l'Amérique du Nord : ainsi la mythologie de la pirogue amazonienne est juxtaposée (à l'exemple du troisième volume des Mythologiques) à celle du passeur menomini (chapitre II), les beuveries cérémonielles du Chaco aux jeûnes initiatiques du Grand Nord (chapitre V) et le cannibalisme tupinamba à la torture iroquoise (chapitre XI). La seule population sud-américaine présentée et analysée de façon indépendante est celle des Araweté (chapitre X), cela dans le cadre d'une critique de From the ennemy's point of view (1992) d'Eduardo Viveiros de Castro, ouvrage qui représente à plusieurs égards une référence majeure pour Désveaux et dont il faut connaître les thèses principales pour comprendre certains arguments de ce dernier.

N'étant pas nous-mêmes américaniste, la discussion que nous mènerons à propos de ces analyses portera sur la méthode et les conclusions théoriques. La critique de leur bien-fondé empirique revient évidemment aux spécialistes des 
régions évoquées ; lesquels, en tout cas, profiteront de revoir leurs terrains dans une perspective nouvelle, parfois osée.

La première hypothèse de ce livre, qu'on aurait souhaité plus amplement et plus explicitement justifiée qu'elle ne l'est, consiste à assigner à la pensée amérindienne une autonomie totale : indépendante tant de la mémoire que des conditions extérieures, elle ne serait sujette qu'aux seules contraintes logiques qui, en revanche, s'imposeraient ici de façon beaucoup plus forte qu'en d'autres régions du monde. Par cette hypothèse, la méthode des transformations, qui rend possible le traitement des systèmes symboliques (du moins dans un premier temps) indépendamment de l'évolution historique et de l'environnement naturel, devient l'instrument universel de l'analyse anthropologique. Les analyses lévistraussiennes ont été principalement centrées sur le mythe considéré comme une construction privilégiée d'une pensée « pure » qui, en opérant au niveau pleinement imaginaire, pouvait se permettre de négliger les nécessités physiques ou sociales (Le Cru et le cuit, 1964, p. 18). Pour Désveaux, la particularité du continent américain consiste précisément dans le fait que la logique transformationnelle imprégnerait toutes les formes symboliques (p. 281) : non seulement le mythe et le rite, mais également l'organisation sociale et la culture matérielle, voire le mode de production et le choix du milieu naturel. Bien plus, et tout en soulignant que cette isomorphie englobante n'implique pas la primauté du mythe sur les autres systèmes qu'il faut envisager comme autonomes (p. 65), il leur dénie toute fonctionnalité particulière, voire toute fonctionnalité autre que celle de la seule cohérence sémantique (pp. 19, 554). Là où Lévi-Strauss avait mis en parallèle les mythes et les organisations sociales (en l'occurrence les structures de parenté) en disant que les deux cherchent à résoudre des problèmes, socio-logiques dans un cas, sociologiques dans l'autre (L'Origine des manières de table, 1968, p. 187), Désveaux avance la thèse que tous les problèmes que se pose la pensée amérindienne sont de nature socio-logique et tout ce qu'on aurait jugé comme servant à l'adaptation aux conditions environnementales ou au fonctionnement interne du corps social n'a en réalité pour objectif que l'équilibre d'un système sémantique.

On peut déplorer que l'auteur, dont la conviction en faveur de cet antifonctionnalisme américaniste est claire, n'ait pas fait plus d'efforts pour en persuader ses lecteurs. Pour cela, il aurait fallu démontrer, premièrement, que la prise en compte des fonctions écologiques ou sociologiques n'apporte rien pour expliquer pourquoi une société a opté pour une certaine transformation plutôt que pour une autre et, deuxièmement, que la même méthode d'analyse donnerait des résultats négatifs si on l'appliquait à d'autres régions du monde (et, surtout, à celles voisines comme la Sibérie). Les quelques paragraphes dans lesquels Désveaux aborde des arguments allant dans ce sens (tels que l'absence d'agriculture en Californie malgré des conditions environnementales identiques à l'aire Pueblo 
(p. 609) ou encore l'affaiblissement successif des processus transformationnels quand on s'éloigne de la Sibérie orientale vers l'Asie centrale (p. 453) - thèses qu'il aurait été intéressant de confronter à celles que Michael Oppitz ${ }^{1}$ émet à propos des transformations de tambours dans cette région) suffisent à montrer que l'auteur détient de nombreux éléments pour mener ce débat, mais ceux fournis restent trop succincts pour étayer la conclusion retenue.

Heureusement, l'adoption de l'idéologie anti-fonctionnaliste n'est nullement indispensable pour accepter la thèse selon laquelle la logique transformationnelle (peut-être pas de façon exclusive) est à l'œuvre dans tous les domaines de la vie sociale en Amérique (et peut-être ailleurs). Cette thèse, maintes fois suggérée mais jamais systématiquement défendue par Lévi-Strauss, est démontrée de façon largement convaincante, particulièrement en ce qui concerne la culture matérielle, dans cet ouvrage. Ce résultat constitue en soi une contribution importante pour l'anthropologie comparative américaniste. Mais celle-ci n'est pas l'objectif de ce livre : l'auteur s'en sert comme d'un pilier pour élargir le cadre de recherche au-delà du mythe afin d'identifier les axes porteurs du système sémantique qui caractérise la pensée amérindienne.

Sans vouloir synthétiser en quelques phrases les conclusions de ce travail de 600 pages, nous proposons de commenter; à titre d'exemple, deux éléments de l'architecture logique de l'Amérique pour lesquels l'auteur montre un fort intérêt. D'une part, l'opposition entre deux directions spatiales - verticale (de la terre au ciel) et horizontale (du près au loin) - que Lévi-Strauss a mise en évidence dans L'Origine des manières de table (1968, p. 154) en montrant que, dans les mythes, la première est associée à l'art culinaire, la seconde à la navigation (tout comme le feu de cuisine lie la terre au ciel, le bateau relie le près au loin). D'autre part, l'opposition entre deux relations sociales - filiation et alliance - qui, cette fois contrairement à Lévi-Strauss, ne sont pas conçues comme existant entre deux groupes sociaux, mais plutôt entre un groupe social et le domaine présocial de la nature brute - domaine où les humains sont mis au monde avant d'être affiliés au groupe et où ils apparaissent comme des voisins avant de devenir des alliés.

Ces deux paires d'opposition - verticale $v$. horizontale et filiation vs. alliance - sont à leur tour associées l'une à l'autre, comme c'est le cas dans le grand mythe américain du " dénicheur d'oiseaux » qui est, rappelons-le, le mythe de référence des Mythologiques et auquel Désveaux consacre deux chapitres. Précisons toutefois que, contrairement à ce que l'on pourrait parfois nous laisser penser dans ce texte (pp. 93, 146, 241), cette association récurrente entre les deux oppositions n'entraîne aucune corrélation terme à terme entre les relations ainsi opposées. Ce n'est pas une similarité entre relations spatiales et relations sociales qui sous-tend la corrélation, mais l'isomorphie du système spatial et du système social; deux systèmes qui résultent, à chaque fois, de l'opposition entre deux relations binaires et qui consistent donc, chacun, en quatre termes pour lesquels quatre permutations (formant un groupe de Klein) sont possibles sans détruire la 
structure : voilà la « quadrature » qu'évoque le titre. Chaque société trouve sa place dans ce quadrant spatial et social en fonction de la manière dont elle met ou non l'accent soit sur les relations au ciel ou au loin, soit sur celles aux fils ou aux beaux-frères.

Illustrons ce propos en commençant par le quadrant spatial tel qu'il est appliqué à la culture matérielle (notamment à l'architecture). Si, sur la Côte Nord-Ouest, on oppose de façon marquée la verticalité (mats sculptés) à l'horizontalité (canots), les Indiens des Plaines combinent les deux directions (par l'obliquité des tipis) tout en diminuant l'opposition entre les deux pôles et, dans l'aire algonkin, on fait disparaître cette opposition (par la forme hémisphérique du wigwam) en la réduisant au degré zéro : tandis que, sur la Côte Nord-Ouest, sont accentués les mouvements vers le ciel et à travers la mer, le wigwam algonkin - nid d'oiseau par sa forme et canot d'écorce par son matériel - symbolise l'immobilisation dans les deux directions, et la tente tremblante en représente le cas limite (pp. 93, 224, 567). Entre ces deux extrêmes, il existe toutes sortes de combinaisons à condition qu'elles soutiennent le symbolisme des directions et ne permettent pas, par exemple, qu'un seul et même objet symbolise une direction par sa forme et une autre par son mouvement - ce qui restreint, entre autres, les objets cylindriques au mouvement axial (à l'instar des canots) et expliquerait ainsi l'absence de la roue en Amérique précolombienne (p. 228). Pour le quadrant social, nous trouvons le même principe de localisation des sociétés : certaines (comme les « tribelets » californiens) font de la filiation l'axe privilégié pour le recrutement dans le groupe social et considèrent l'alliance comme une nécessité physique comparable à l'alimentation (les femmes étant assimilées aux gibiers) (p. 602). D'autres (comme les sociétés claniques de l'Est) mettent l'accent sur l'alliance et réduisent la filiation à la naissance physique, ce qui explique le paradigme " totémique » de la reproduction des animaux et la place centrale qu'occupent les mères dans la formation du « clan ». Détail important dans ce contexte : la pensée amérindienne attribuerait la mise au monde "naturelle» exclusivement aux femmes, alors que la filiation proprement dite serait une affaire purement masculine et ne permettrait pas de constituer des clans au sens strict, mais des confréries fondées sur la cooptation et pas sur la naissance - les « patriclans » apparents, e.g. parmi les Sioux orientaux, ne seraient en fait que des sociétés secrètes transformées (p. 160 passim, p. 584). L'opposition entre filiation et alliance - opposition selon laquelle se distinguent les deux types de sociétés évoqués plus haut - se rattache donc à la différence entre les sexes. D'autres sociétés encore (parmi lesquelles celle des Inuit est la plus représentative) se servent de l'alternance saisonnière pour opposer un état estival de la société - perçue en tant que communauté de naissance et de co-résidence, dirigée par les femmes, et dont tous les liens constitutifs ont été réduits aux liens « naturels » - à un état hivernal - dans lequel la filiation et l'alliance, cérémoniellement manipulées par les hommes, sont considérées comme complètement indépendantes de la 
nature et re-fondent la société en une grande confrérie endogame (p. 409). Chaque société présente donc une combinaison unique des deux manières d'opposer la culture à la nature, le libre choix des membres du groupe et de leurs alliés à la contrainte physique de la descendance et du partage des ressources naturelles, la confrérie et l'unité endogame au clan et au groupe résidentiel (p. 320). Tout comme dans le cas de la morphologie spatiale, toutes les formes intermédiaires peuvent être imaginées à la seule condition que la valeur distinctive des institutions ne se perde pas : l'acte de filiation ne peut pas redoubler la mise au monde, l'alliance de mariage ne peut pas être nouée entre des personnes qui résident ensemble (p. 312 passim). Principe qui, au bout du compte, rend contradictoires des notions comme « filiation matrilinéaire » ou « endogamie locale » en Amérique : dans les sociétés qui considèrent la filiation et l'alliance comme fondamentales pour leur constitution, les fils idéaux seraient des non-consanguins (c'est-àdire des adoptés), les beaux-frères idéaux seraient les étrangers (c'est-à-dire les ennemis).

Nous reviendrons plus loin sur cette thèse cruciale, dont le fondement hormis pour certains cas spéciaux comme les Dakota ou les Jivaro - nous semble néanmoins plus faible que celui des analyses concernant la culture matérielle. L'auteur ne donne aucune définition du groupe de filiation et du groupe résidentiel, ce qui nourrit le soupçon qu'il les définit justement par l'absence ou, du moins, par l'insignifiance des liens consanguins et des mariages locaux : une filiation consanguine (et surtout utérine) ne serait pas une filiation proprement dite, une alliance entre co-résidents ne serait pas une vraie alliance. L'argument devient alors circulaire. En effet, on peut difficilement se défendre de l'impression qu'il aurait été développé à l'extérieur de l'analyse comparative des organisations sociales et tiendrait moins de la sociologie que de l'idéologie des Amérindiens, telle qu'elle ressort dans l'analyse des mythes. Idéologie qui les empêcherait de penser la filiation et l'alliance comme des liens entre deux parties égales d'un même groupe social, mais qui en fait plutôt deux relations liant le groupe social à son extérieur. On verra que cette contrainte idéologique est une contrainte logique imposée, selon l'auteur, par la pensée transformationnelle même.

Si le continent américain constitue, comme tout dans ce livre tend à le montrer, « un vaste système en état d'équilibre homothétique logique » (p. 21) - ou, pour le dire autrement, si chaque culture, située en un lieu et à un moment donnés sur ce continent, ne représente dans sa totalité qu'un certain état transformationnel d'une seule et même structure sémantique -, cette convertibilité universelle de toute culture en toute autre ne peut évidemment pas rester sans conséquence pour les relations d'une culture, soit à la culture qui la précède, soit aux cultures qui l'entourent. Une transformation étant par définition une application bijective - donc réversible -, aucune culture ne peut contenir un surplus 
que l'autre ne saurait assimiler : tout ce qu'elle représente était déjà préexistant sous une autre forme, tout ce qu'elle voit chez ses voisins ne lui fournit que sa propre image en miroir. Incapables de comprendre la moindre sorte de nouveauté dans le temps et d'altérité dans l'espace, les cultures amérindiennes resteraient sans histoire et sans étranger. Ni le passé, ni l'Autre ne pourraient leur apprendre quelque chose. Foncièrement ahistoriques et ethnocentriques, elles nieraient l'existence de tout ce qu'elles ne parviendraient pas, par une série de transformations adéquates, à retrouver chez elles. De par leur constitution transformationnelle, les sociétés américaines resteraient ainsi non seulement « froides » au sens de Lévi-Strauss, mais aussi xénophobes :

[...] La principale caractéristique de la logique transformationnelle [...] réside dans sa transitivité intrinsèque, synonyme de transparence. En vertu de cette convertibilité absolue, un groupe quel qu'il soit se trouve dans l'incapacité de percevoir la culture d'un groupe voisin comme différente de la sienne dans la mesure où elle lui est totalement intelligible. Il ne s'agira jamais pour lui que d'une série d'inversions plus ou moins accentuées de ses propres références mythiques, rituelles ou sociologiques [...]. Du coup, [le système sémantique] interdit l'émergence d'une quelconque altérité parmi les humains, reportant celle-ci au-delà de la barrière du langage ordinaire, du côté des animaux ou des esprits. (p. 279)

Cette manière de caractériser le rôle de l'altérité dans la pensée amérindienne s'oppose nettement à ce que les travaux de Lévi-Strauss et, plus récemment, ceux de Viveiros de Castro nous ont habitués à lui associer. Selon ces auteurs, le principe organisateur même de l'opposition binaire ne permet pas aux Amérindiens de penser une totalité composée de deux termes opposés l'un à l'autre, sans l'opposer à son tour à un troisième terme, et ainsi de suite à l'infini. Cette dynamique d'engendrement d'une série de termes de plus en plus éloignés du terme de référence s'appliquerait également à la façon dont une société se pense : comme Lévi-Strauss l'a illustré avec l'exemple d'un mythe tupinamba dans Histoire de lynx (1991, p. 76), l'opposition intérieure entre deux moitiés de la société ne serait pas pensable sans l'opposition extérieure entre la société tout entière et les étrangers, tout comme cette dernière impliquerait d'emblée celle entre les Indiens (concitoyens et étrangers ensemble) et les Blancs - ce qui rendrait compte de l'extraordinaire simplicité avec laquelle toutes les sociétés amérindiennes, du Brésil jusqu'en Colombie Britannique, ont su intégrer l'apparition des Européens dans leur mythologie (ibid., p. 292). Selon cette logique, la convertibilité totale entre les cultures qu'impose la pensée transformationnelle fournirait le cadre de l'intégration successive d'une série illimitée de cultures de différents degrés d'altérité - étant entendu que cette altérité ne concerne pas la convertibilité en tant que telle (à cet égard, les Bororo seraient aussi proches des Klamath que des Gé), mais la hiérarchie sémantique des oppositions par lesquelles s'opère la conversion. 
À la différence de cette approche classique, Désveaux définit l'altérité par la non-convertibilité - la meilleure définition d'un groupe social serait celle de " la somme des individus qui partagent une même mythologie et une même nomenclature (ou plus exactement l'espace d'acceptation des variantes du mythe et de la nomenclature) » (p. 500) - et exclut, en revanche, la possibilité de toute hiérarchie du proche et du lointain au sein d'un même système d'oppositions : les systèmes symboliques amérindiens seraient caractérisés par "l'occlusion de la dualité » (p. 373), sans qu'une totalité faite de deux éléments opposables ait encore besoin de s'opposer à un troisième terme qui - affirmation dirigée explicitement contre Viveiros de Castro - ne serait qu'une illusion en Amérique (p. 230). L'étranger n'a pas de place dans le système d'oppositions, il ne désigne que ce qui est hors système, il ne s'oppose à aucun terme en particulier, mais à l'ensemble des termes : l'étranger est celui qui n'a pas de nom (p. 255) et avec lequel on ne parle pas (p. 257). Tant qu'il y a communication, il n'y a pas d'Autre.

$\mathrm{Si}$ on combine cette thèse avec la présupposition que le continent américain tout entier forme un vaste système de transformations, la conclusion semble donc s'imposer : les populations indiennes de ce continent ne constitueraient qu'un seul et même groupe social dont les membres se sentiraient également proches les uns des autres. Or rien n'est plus éloigné de la position de l'auteur qu'une telle affirmation! Bien au contraire, l'horizon d'« acceptation des variantes » serait extrêmement étroit en Amérique, où chaque groupe social constituerait un îlot dans un vaste univers d'étrangers - animaux, esprits, non-humains par définition. Dépourvu d'existence symbolique aussi bien que sociale, réduit à l'état de nature brute, cet environnement d'étrangers n'a aucune communication réciproque avec la société et ne lui sert que comme ressource - ressource alimentaire bien sûr, mais aussi ressource pour la filiation et l'alliance qui ne sont finalement qu'un autre type d'appropriation (pp. $158 \mathrm{sq}$.). Vues sous cet angle, la guerre, la chasse aux têtes, l'anthropophagie, la torture ne correspondent plus à des moyens pour anéantir l'Autre et l'expulser de la culture, mais bien au contraire pour l'arracher à la nature et l'intégrer dans le système culturel (pp. 276, 394). L'acte prédateur devient ainsi le paradigme de la reproduction culturelle par filiation et alliance : le fils idéal est l'adopté (p. 157), l'épouse idéale, la captive (p. 285), le beau-frère idéal, l'ennemi qu'on tue, torture et dévore (p. 279).

Pour caractériser la filiation et l'alliance en tant qu'actes reproducteurs de la culture, il ne suffit pas de dire qu'ils s'opposent à la reproduction naturelle, que la filiation est presque par définition exogène par rapport à la consanguinité, de même que l'alliance est exogame par rapport à la communauté d'alimentation. La réalisation de ces actes culturels présuppose tout de même ce réservoir naturel de fils et d'épouses, lesquels ne sont pas, du moins idéalement, recrutés dans un autre groupe reconnu : la filiation et l'alliance ne sont pas des transactions entre groupes sociaux. En particulier, «l'Amérique reste largement, sinon totalement, opaque face à l'heuristique de l'échange matrimonial » (p. 618) et si 
les nomenclatures de parenté font partie d'un système transformationnel, elles n'ont pas pour but de régler les mariages. Ainsi la terminologie dravidienne qui, selon la tradition structuraliste, est l'expression directe du mariage à échange, ne représente pour Désveaux qu'un système symbolique de dualisme égocentré opérant par l'imposition d'une équivalence entre affinité et collatéralité (pp. 513, 534). De la même façon, tandis que Lévi-Strauss, dans Anthropologie structurale (1958, p. 263), avait interprété, à l'aide justement des mythes et des rites, les systèmes Crow-Omaha comme des organisations à alliance matrimoniale fondées sur une équivalence entre les deux oppositions père/fils et mari/épouse, ce qui lui avait permis d'assimiler la filiation rituelle à l'échange des femmes (par exemple, chez les Mandan, les fils prêtent leurs épouses à leurs pères cérémoniels), Désveaux les détache nettement de toute connotation matrimoniale en les fondant (de façon assez proche de celle de Françoise Héritier dont il se démarque pourtant) sur une équivalence entre la différence de générations, la dichotomie aîné/cadet et la différence sexuelle (pp. 510, 515).

Cette mise à distance des terminologies de parenté par rapport à tout ce qui pourrait sembler régler les alliances (et les filiations) n'est qu'une conséquence logique de la thèse selon laquelle l'alliance et la filiation lient le groupe social au groupe de ceux qui n'ont pas de nom. Axes centraux autour desquels s'organisent tous les systèmes symboliques - mythe, rite, organisation sociale -, ces deux relations s'établissent précisément entre le système symbolique et son extérieur muet, cet horizon du non symbolique et du non communicable que les mythes décrivent, tantôt comme région céleste, tantôt comme région outre-mer, selon qu'ils avancent la conquête du feu ou la maîtrise des eaux comme paradigme de l'appropriation de la nature par la culture. À la différence de toutes les oppositions binaires qui s'établissent entre les innombrables formes de cette relation primordiale - alliance $v s$. filiation, horizontalité $v$ s. verticalité, etc. -, son contenu même, ultime signifié de tous les systèmes symboliques, n'entre pas dans le jeu des transformations réciproques. Aucune transformation ne peut établir une convertibilité entre culture et nature, entre les humains et les non-humains, entre le groupe et ses ennemis. Et, pourtant, ces non-humains, dépourvus de tout système symbolique aux yeux de leurs voisins, posséderaient, pour l'anthropologue, un système symbolique non seulement équivalent, mais totalement convertible en celui justement de leurs voisins.

Voilà donc la contradiction principale à laquelle on se heurte tout au long de cette discussion : comment une convertibilité totale entre deux sociétés pourraitelle ne pas être remarquée par les intéressés eux-mêmes, voire être imaginable sans un échange culturel continu et intensif ? Comment une culture pourrait-elle reléguer à l'état de nature toute autre culture qui, selon les mots de l'auteur, lui devrait être quand même " totalement intelligible»? Il ne semble y avoir que deux solutions à ce dilemme : ou bien on conçoit la relation entre culture et nature comme une relation réciproque en attribuant à ces deux termes une valeur 
purement déictique, comme le propose Viveiros de Castro ; ou bien - et ceci est la solution proposée dans ce livre - on détache la notion de transformation de tout rapport réel de communication et on en fait une relation purement idéelle, une similarité de structure qui s'expliquerait par le fait que chaque culture évoluerait sous les mêmes contraintes internes, indépendamment de toute autre. En tournant ainsi les sociétés amérindiennes en monades sans fenêtres, Désveaux parvient à réconcilier les deux images contradictoires qu'il dessine de ce continent : celle d'une harmonie universelle qui fait correspondre toute société à toute autre et celle d'une guerre de tous contre tous qui enferme chaque société dans son monde subjectif.

Nous tournant finalement vers l'aspect formel des transformations, auquel ce livre accorde une grande importance (y compris un chapitre à part), ce n'est pas pour ouvrir une nouvelle discussion qui n'aurait rien à voir avec les réflexions précédentes. Étant donné l'énorme masse des combinaisons possibles qui résultent d'un petit inventaire de termes et de relations et qui donneraient une latitude presque hors contrôle à la recherche des analogies, inversions et équivalences de toutes sortes, l'analyse transformationnelle aura toujours du mal à se démarquer d'un jeu arbitraire sans l'appui d'une méthode suffisamment rigide et d'un symbolisme suffisamment précis pour rendre possible la contre-épreuve de ses hypothèses. Toute démonstration d'un rapport transformationnel, aussi convaincante soit-elle, restera une performance artistique, plutôt que scientifique, tant on aurait pu avec la même élégance démontrer n'importe quel autre rapport. En fait, les rapports transformationnels n'ont rien d'étonnant en soi ; il serait bien plus étonnant de ne pas trouver de similarités structurelles - fussent-elles purement aléatoires - dans un corpus aussi dense et différencié qu'est l'ethnographie de l'Amérique.

Or; dans les Mythologiques de Lévi-Strauss, le postulat n'est pas qu'il est possible d'établir des transformations entre les mythes (ce qui est presque une trivialité), mais que ces transformations forment un groupe agissant sur l'ensemble des mythes. Ce qui implique tout d'abord que la même transformation qui convertit un mythe $A$ en un mythe $B$ doit aussi convertir tout mythe $C$ en un quelconque mythe $D$. La possibilité de trouver ce mythe $D$ constitue une première épreuve empirique pour savoir si l'on a correctement reconstruit la transformation en question, et la précision avec laquelle ce mythe recherché peut être décrit à l'avance - autrement dit, la diffículté à se débrouiller avec n'importe quel mythe à la main - est une mesure de scientificité de l'analyse. Si le chemin commencé par Lévi-Strauss doit aboutir un jour à une véritable science des transformations symboliques - ce qui implique la possibilité de reproduire tous les résultats de l'analyse transformationnelle dans une série de pas explicites et compréhensibles -, il est évident que la base formelle d'une telle science prendra appui sur la théorie des groupes. Lévi-Strauss avait déjà indiqué cette orientation en se 
servant, ne serait-ce qu'à des fins d'illustrations, du plus simple groupe de transformations symétriques qu'on puisse imaginer au-delà des groupes triviaux (d'ordre 1 ou 2), à savoir le groupe dit « de Klein », groupe d'ordre 4 engendré par deux transformations de période 2 (autrement dit par deux oppositions).

En revanche, l'autre célèbre formalisme utilisé par Lévi-Strauss pour illustrer ses réflexions - la « formule canonique »-semble encore beaucoup plus proche de la magie que de la mathématique. S'il est certain que la transformation qu'elle suggère ne peut pas faire partie d'un groupe de Klein puisqu'elle n'est pas symétrique - liant le terme $a$ au terme $b$, elle lie le terme $b$ à un troisième terme $a^{-1}$ (plutôt qu'au premier terme $a$ ) -, la métamorphose de ce troisième terme en une fonction constitue jusqu'à présent une opération sans aucun sens précis, ce qui rend l'application de la formule canonique aussi facile que gratuite. Les quelques efforts exégétiques pour lui donner après coup un sens mathématique (au lieu de trouver un meilleur formalisme pour exprimer le rapport établi par Lévi-Strauss) n'ont pas donné des résultats très utiles : certains l'interprètent comme chiffre d'un groupe non commutatif, d'autres comme une articulation entre deux groupes, sans toutefois appliquer cet appareil mathématique aux matériaux ethnographiques (plutôt qu'à l'œuvre lévi-straussienne). On est donc très intéressé quand Désveaux propose « une méthode qui permette de convertir le groupe de Klein en formule canonique et réciproquement » (p. 45) et cela non seulement pour interpréter Lévi-Strauss, mais pour se construire un vrai instrument de travail qu'il emploiera systématiquement au cours des analyses concrètes.

Cependant, la comparaison faite entre les dix-huit exemples d'application de la formule dans ce livre ne permet pas d'en tirer une quelconque méthode constante et reproductible. Si les groupes de Klein que construit l'auteur s'avèrent être des instruments valables de l'analyse et servent effectivement à rendre explicites les rapports mutuels des transformations qui sont en jeu, leur conversion en formules canoniques reste une opération supplémentaire dont on ne saisit ni le principe, ni l'utilité. Elle consiste à distribuer parmi les cinq variables de la formule lévi-straussienne ( $a, b, a^{-1}, x$ et $\left.y\right)$, soit les quatre éléments de l'ensemble sur lequel agit le groupe de transformations, soit les quatre marques distinctives (opposées deux à deux) qui caractérisent ses deux transformations génératrices, plus un cinquième élément jugé représenter la structure dans sa totalité et, parfois, d'autres éléments supplémentaires qui se substituent aux éléments abandonnés du groupe initial. Cette distribution se fait de façon complètement arbitraire (le cinquième élément se trouvera dans les cinq positions possibles de la formule canonique ; il est en outre employé dans cinq variantes différentes). Enfin, aucune règle n'est donnée (ou même n'apparaît implicitement) sur la façon de lire la formule résultante, ce qui implique, au bout de compte, que n'importe quelle constellation de symboles peut avoir une interprétation plus ou moins plausible, et n'importe quel résultat souhaité peut être « déduit » au moyen de la formule canonique. 
On aurait souhaité que l'auteur se limite à l'instrument fertile des groupes de Klein - voire peut-être à d'autres groupes plus compliqués comme ceux à huit éléments (dont certains ne sont plus commutatifs) - et oublie ce lourd appareil de formules qui brouille le lecteur au lieu de lui servir de repère. Qui plus est, comme leur interprétation exige souvent des arguments ad hoc, l'emploi de ces formules, sans rien apporter de plus à l'analyse transformationnelle, risque de la discréditer comme un jeu postmoderniste, ce qui ferait du tort non seulement à cet ouvrage rigoureux, mais à tout le programme scientifique qu'il représente et qu'il cherche à avancer dans un environnement encore largement sceptique face aux analyses de cette sorte. Cela est d'autant plus vrai que ce livre présente des résultats beaucoup plus généraux et radicaux que Lévi-Strauss ne les avait jamais envisagés. Le panlogisme des sociétés amérindiennes, le binarisme fermé de leur pensée, l'impossibilité de l'altérité, l'universalité du paradigme de la prédation, l'absence de l'alliance réciproque, l'opposition entre filiation et consanguinité, la différence fondamentale entre parenté agnatique et utérine - autant de thèses peu communes qui, pour vraiment convaincre, auront besoin d'un appui plus ample et plus fort que cet essai ne le peut fournir.

Mais on ne rendrait pas justice à ce livre si on le prenait, contre l'avis de l'auteur (p. 14), comme une œuvre accomplie plutôt que comme une importante étude exploratoire, préliminaire à un projet de recherche plus vaste qui exigera, sinon " plusieurs vies » comme le reconnaît l'auteur, du moins plusieurs mains, et probablement quelques ordinateurs. En effet, une analyse systématique de la structure relationnelle d'un grand corpus de systèmes symboliques amérindiens, même limités à un seul domaine (par exemple la culture matérielle ou la terminologie de parenté), n'est pas imaginable sans l'aide de l'informatique. Les problèmes attachés à une telle entreprise, en commençant par le codage, sont nombreux, mais moins insurmontables qu'il y a 50 ans quand Lévi-Strauss déplorait encore son impossibilité technique. Or, si ce livre fait bien ressortir la problématique d'une analyse transformationnelle en forme d'essai et de la main d'un seul individu, il ne laisse aucun doute sur les compétences d'Emmanuel Désveaux à poursuivre un tel projet. Témoignant d'un horizon ethnographique étendu, d'une grande sensibilité pour ce qui est des rapports d'isomorphie entre systèmes symboliques apparemment hétérogènes et d'une conscience de la valeur des méthodes formelles dans ce genre de recherches, cette Quadratura Americana laisse espérer l'ouverture d'une nouvelle étape dans le développement de l'anthropologie transformationnelle.

1. OppIтz Michael, 1999, « Materielle Kultur und Transformationsbegriff. Zur Morphologie der Schamanentrommel ", in Symbol-Struktur - Kultur. Zur erkenntnistheoretischen Grumdlegung der Sozial- und Kulturwissenschaften nach Ernst Cassirer, Claude Lévi-Strauss und Pierre Bourdieu, Mitteilungen des Instituts für Wissenschaft und Kunst, Jahrgang, 54, pp. 27-40. 